\title{
Reference Tracking MPC using Terminal Set Scaling
}

Daniel Simon, Johan Löfberg and Torkel Glad

\section{Linköping University Post Print}

N.B.: When citing this work, cite the original article.

Original Publication:

Daniel Simon, Johan Löfberg and Torkel Glad, Reference Tracking MPC using Terminal Set Scaling, 2012, Proceedings of the 51st IEEE Conference on Decision and Control, 4543-4548, 978-1-4673-2065-8.

http://dx.doi.org/10.1109/CDC.2012.6426550

Copyright: IEEE

Postprint available at: Linköping University Electronic Press

http://urn.kb.se/resolve?urn=urn:nbn:se:liu:diva-89481 


\title{
Reference Tracking MPC using Terminal Set Scaling
}

\author{
Daniel Simon Johan Löfberg Torkel Glad \\ Departement of Electrical Engineering \\ Linköping University \\ SE-581 83 Linköping, Sweden
}

\begin{abstract}
A common assumption when proving stability of linear MPC algorithms for tracking applications is to assume that the desired setpoint is located far into the interior of the feasible set. The reason for this is that the terminal state constraint set which is centered around the setpoint must be contained within the feasible set. In many applications this assumption can be severely limiting since the terminal set is relatively large and therefore limits how close the setpoint can be to the boundary of the feasible set. We present simple modifications that can be performed in order to guarantee stability and convergence to setpoints located arbitrarily close to the boundary of the feasible set. The main idea is to introduce a scaling variable which dynamically scales the terminal state constraint set and therefore allows a setpoint to be located arbitrarily close to the boundary. In addition to this the concept of pseudo setpoints is used to gain the maximum possible region of attraction and to handle infeasible references. Recursive feasibility and convergence to the desired setpoint, or its closest feasible alternative, is proven and a motivating example of controlling an agile fighter aircraft is given.
\end{abstract}

\section{INTRODUCTION}

Input and state constrained optimal control problems are in many cases impossible to solve with an explicit feedback policy [1]. Model Predictive Control (MPC) offers a way to solve these problems by iteratively solving a constrained optimization problem online, using the current state of the system as initial conditions.

Due to the iterative nature of MPC one must take special measures to ensure that the optimization problem remains feasible and stabilize the system. However these measures can in the severe cases limit the reference tracking ability of the controller to a small region around the origin.

The main goal of this article is to improve the possibility of reference tracking in linear MPC by adjusting the existing stability measures so that they are more suitable for tracking.

In section II an introduciton to the MPC formulation and the stability measures are outlined. We present the modifications suggested for the reference tracking application and the main theorem of the paper in section III. In section IV the proof of theorem 1 is presented. The article finishes with an example from the aircraft industry in section $\mathrm{V}$.

\section{MPC AND STABILITY}

Among the many different formulations of Model Predictive Control (MPC) with guaranteed stability, the one that has attracted most attention and is most widely used (at least in academia) is the formulation with a terminal cost and constraint [1]. This is also referred to as the dual mode formulation since, theoretically, the objective of the MPC controller is to steer the system state to a region around the origin (terminal set) where a local feedback policy takes over.

For the discrete time system

$$
x_{k+1}=f\left(x_{k}, u_{k}\right)
$$

with state constraints $x_{k} \in \mathcal{X}$ and control constraints $u_{k} \in$ $\mathcal{U}$, the MPC control law is defined through the solution of a finite horizon optimization problem with stage cost $\ell\left(x_{k}, u_{k}\right)$, terminal state penalty $\Psi\left(x_{k+N}\right)$ and a terminal state constraint $\mathcal{T}$.

$$
\begin{aligned}
\min _{u} . & \Psi\left(x_{k+N}\right)+\sum_{i=0}^{N-1} \ell\left(x_{k+i}, u_{k+i}\right) \\
\text { s.t. } & \\
x_{k+i+1}= & f\left(x_{k+i}, u_{k+i}\right) \\
x_{k+i} & \in \mathcal{X} \\
u_{k+i} & \in \mathcal{U} \\
x_{k+N} & \in \mathcal{T}
\end{aligned}
$$

Classical stability results for MPC essentially states that if $\mathcal{T} \subseteq \mathcal{X}$ is a positively invariant set (see Definition 3 ) of the system (1) controlled with the feedback $u_{k}=\kappa\left(x_{k}\right)$ where $\kappa\left(x_{k}\right) \in \mathcal{U} \forall x_{k} \in \mathcal{T}, \ell\left(x_{k}, u_{k}\right)$ is positive definite, and $\Psi$ is chosen to be a Lyapunov function upper bounding the infinite horizon cost when using the controller $\kappa\left(x_{k}\right)$, then the closed loop system is stable and asymptotically converges to the origin [1].

Our goal is to make minimal changes to this framework, for the linear polytopic case, in order to develop a flexible reference tracking algorithm with guaranteed stability, which is intuitive and easily understood.

\section{A. Reference tracking}

When tracking a reference signal, i.e., the so called servo problem, the system shall not converge to the origin but settle at some steady state $\left(x_{r}, u_{r}\right)$ different from the origin.

At steady state it must hold that $x_{k+1}=x_{k}=x_{r}$ and $y_{k}=r$. Given a controllable linear discrete time system

$$
\begin{aligned}
x_{k+1} & =A x_{k}+B u_{k} \\
y_{k} & =C x_{k}+D u_{k}
\end{aligned}
$$

we must first select a combination of input and steady state, yielding the desired output. If the choice is non-unique, a 
reasonable choice is the minimal norm input, which can be formulated as a quadratic program, [2]

$$
\begin{gathered}
\min _{x_{r}, u_{r}} u_{r}^{T} R_{s} u_{r} \\
\text { s.t. } \\
{\left[\begin{array}{cc}
A-I & B \\
C & D
\end{array}\right]\left[\begin{array}{l}
x_{r} \\
u_{r}
\end{array}\right]=\left[\begin{array}{l}
0 \\
r
\end{array}\right]} \\
u_{\min } \leq u_{r} \leq u_{\max }
\end{gathered}
$$

A pragmatic approach to implement the reference tracking case is to simply shift the origin of the problem and apply the standard MPC scheme on the translated system. i.e., in the original coordinate system, penalize deviations from the steady state setpoints, and shift the terminal set such that it is centered at the steady state.

$$
\begin{aligned}
\min _{u} \Psi\left(x_{k+N}-x_{r}\right)+ & \sum_{i=0}^{N-1} \ell\left(x_{k+i}-x_{r}, u_{k+i}-u_{r}\right) \\
\text { s.t. } & \\
x_{k+i+1} & =f\left(x_{k+i}, u_{k+i}\right) \\
x_{k+i} & \in \mathcal{X} \\
u_{k+i} & \in \mathcal{U} \\
x_{k+N} & \in \mathcal{T}\left(x_{r}\right)
\end{aligned}
$$

To represent translation of a set, we used the notation $\mathcal{T}\left(x_{r}\right)=x_{r} \oplus \mathcal{T}$, i.e., the Minkovsky set addition.

This problem formulation is the standard procedure of solving tracking problems in the MPC framework. This standard formulation has in the last couple of years been analyzed and extended to guarantee offset free tracking in the presence of disturbances and model uncertainties [3][9].

A further extension is to use a so called pseudo setpoint [10] or admissable reference [6]. By using this pseudo reference, the feasible region of the problem can be increased. Instead of using the true reference $r$ in (4) one introduces a new variable $\bar{r}$ which gives a corresponding $\bar{x}$ and $\bar{u}$ in the optimization problem (5), and then penalize the deviation between the desired reference $r$ and the pseudo reference $\bar{r}$ using a positive definite function $\phi(\bar{r}-r)$

$$
\min _{u, \bar{r}} \Psi\left(x_{k+N}-\bar{x}\right)+\sum_{i=0}^{N-1} \ell\left(x_{k+i}-\bar{x}, u_{k+i}-\bar{u}\right)+\phi(\bar{r}-r)
$$

$$
\begin{aligned}
\text { s.t. } & \\
x_{k+i+1} & =f\left(x_{k+i}, u_{k+i}\right) \\
x_{k+i} & \in \mathcal{X} \\
u_{k+i} & \in \mathcal{U} \\
x_{k+N} & \in \mathcal{T}(\bar{x})
\end{aligned}
$$

However, crucially and easily missed, in order to guarantee stability it must hold that the translated terminal set still is positively invariant and satisfies constraints. What easily can happen is that $\mathcal{T}(\bar{x}) \nsubseteq \mathcal{X}$, i.e., the translation of $\mathcal{T}$ moves parts of it outside $\mathcal{X}$, thus invalidating any claim of positive invariance (and similarly w.r.t to control constraints for the nominal controller in $\mathcal{T}(\bar{x})$. Hence, if $\mathcal{T}$ is large (which is a good thing when controlling around the origin since it will ensure that the terminal state constraint has little impact on feasibility) it can only be translated a short distance without violating feasibility, thus only allowing a very limited range of steady states $\bar{x}$.

This is a major drawback since many applications have optimal operating points on or close to the border of the feasible set. A method to handle this has been developed in [11]. However, the authors assume that the system has enough degrees of freedom to constrain the evolution of the states along any linear constraint, which is not always the case. This motivates an extension of the theory.

\section{EXTENSION OF TRACKING ALGORITHM}

In the remainder of this paper we will restrict our discussion to discrete time linear systems with polytopic ${ }^{1}$ constraints. Before we derive the extended tracking algorithm let us first state some necessary assumptions, facts and definitions.

Assumption 1: The matrix

$$
\left[\begin{array}{cc}
A-I & B \\
C & D
\end{array}\right]
$$

is such that there exists a minimum norm solution to (4) and the unconstrained solution can be written as a linear mapping from the pseudo reference $\bar{r}$ to the steady state and controls $\bar{x}$ and $\bar{u}$ as

$$
\left[\begin{array}{l}
\bar{x} \\
\bar{u}
\end{array}\right]=\left[\begin{array}{l}
\Pi_{x} \\
\Pi_{u}
\end{array}\right] \bar{r}
$$

Assumption 2: $\mathcal{X}, \mathcal{U}$ and $\mathcal{T}$ contain the origin in their interior.

Definition 1: For a given constant $1>\epsilon>0$ and set $\mathcal{Z}$ containing the origin, let $\operatorname{int}_{\epsilon}(\mathcal{Z})=(1-\epsilon) \mathcal{Z}=$ $\left\{z \mid \frac{1}{1-\epsilon} z \in \mathcal{Z}\right\}$.

Definition 2: For a given $\epsilon$, let $r_{\perp}$ be the closest strictly feasible point to the reference $r$ in a $W$-weighted Euclidean distance measure.

$$
\begin{aligned}
& r_{\perp}=\underset{\bar{r}}{\arg \min }\|\bar{r}-r\|_{W}^{2} \\
& \text { s.t. } \quad \Pi_{x} \bar{r} \in \operatorname{int}_{\epsilon}(\mathcal{X}), \quad \Pi_{u} \bar{r} \in \operatorname{int}_{\epsilon}(\mathcal{U})
\end{aligned}
$$

Definition 3: The set $\mathcal{T} \subset \mathbb{R}^{n}$ is said positively invariant for a system $x_{k+1}=f\left(x_{k}\right)$ if for all $x_{k} \in \mathcal{T}$ the solution $x_{k+i} \in \mathcal{T}$ for $i>0$.

Lemma 1: Let $\mathcal{T}$ be a positively invariant set for the stable linear system $x_{k+1}=A x_{k}$. Then for any scalar $\lambda \geq 0, \lambda \mathcal{T}$ is also a positively invariant set for the system.

Proof: The lemma follows directly from the properties of stable discrete time linear systems, see e.g. [12].

With these definitions and assumption in order, we are ready to formulate the extended tracking algorithm. As a

\footnotetext{
${ }^{1}$ Polytopes are assumed to be a closed and bounded intersection of a finite number of half-spaces.
} 
first step, we give a high-level representation of the problem, which we soon will write as a quadratic program

$$
\begin{aligned}
& \min _{u, \lambda_{k}, \bar{r}_{k}} \mathcal{J}_{k} \\
& \text { s.t. } \\
& x_{k+i+1}=A x_{k+i}+B u_{k+i} \\
& x_{k+i} \in \mathcal{X} \\
& u_{k+i} \in \mathcal{U} \\
& x_{k+N} \in \lambda_{k} \mathcal{T}\left(\bar{x}_{k}\right) \\
& \bar{u}_{k}-K\left(x-\bar{x}_{k}\right) \in \mathcal{U} \forall x \in \lambda_{k} \mathcal{T}\left(\bar{x}_{k}\right) \\
& \lambda_{k} \mathcal{T}\left(\bar{x}_{k}\right) \subseteq \mathcal{X} \\
& \bar{x}_{k}=\Pi_{x} \bar{r}_{k} \in \operatorname{int}_{\epsilon}(\mathcal{X}) \\
& \bar{u}_{k}=\Pi_{u} \bar{r}_{k} \in \operatorname{int}_{\epsilon}(\mathcal{U})
\end{aligned}
$$

where

$$
\begin{aligned}
\mathcal{J}_{k}= & \left\|x_{k+N}-\bar{x}_{k}\right\|_{P}^{2}+\left\|\bar{r}_{k}-r\right\|_{W}^{2} \\
& +\sum_{i=0}^{N-1}\left(\left\|x_{k+i}-\bar{x}_{k}\right\|_{Q}^{2}+\left\|u_{k+i}-\bar{u}_{k}\right\|_{R}^{2}\right)
\end{aligned}
$$

In (8), $Q$ and $R$ are positive definite weight matrices, used also to define the Lyapunov cost matrix $P$ and nominal state feedback $K$ through

$$
(A-B K)^{T} P(A-B K)-P=-Q-K^{T} R K
$$

The matrix $W$ is positive definite, $r$ is the desired reference to track and $\bar{r}_{k}$ is the pseudo reference variable [10]. The set $\mathcal{T}$ is a polytopic positively invariant set for the system (7b) with the local controller $u_{k}=-K x_{k}$ such that all constraints on $x$ and $u$ are satisfied in $\mathcal{T}$. The polytopes $\mathcal{X}$ and $\mathcal{U}$ are the feasible sets of $x$ and $u$ and the matrices $\Pi_{x}$ and $\Pi_{u}$ are defined in Assumption 1. The main new addition here is the scaling variable $\lambda_{k}$. This scaling allows us to move the terminal state set arbitrarily close to the border of the feasible set, since it can be scaled down to a point. The fact that we are constraining $\bar{x}_{k}$ and $\bar{u}_{k}$ to be strictly feasible (i.e., placing the terminal set in the strict interior) is motivated from a theoretical point of view. It is currently required in the stability proof but simulations show that for practical purposes any arbitrarely small $\epsilon$ can be chosen, even $\epsilon=0$.

As the problem is written in (7), it is not suitable for optimization. For instance, (7e) and (7g) are not obviously linear in $\lambda_{k}$ and $\bar{x}_{k}$, and (7f) is an infinite-dimensional constraint. Hence, a reformulation is needed.

Let us begin with (7e) which models that the terminal state is inside the scaled and translated terminal set. Since the terminal state is polytopic, we have a representation of the form $\mathcal{T}=\left\{x \mid F_{\mathcal{T}} x \leq b_{\mathcal{T}}\right\}$. With our definitions of translations and scaling, we obtain the model $F_{\mathcal{T}}\left(x_{k+N}-\right.$ $\left.\bar{x}_{k}\right) \leq \lambda_{k} b_{\mathcal{T}}$, i.e., a linear constraint.

The constraints $(7 \mathrm{~g})$, which ensure the scaled and translated terminal set to be be state feasible, and (7f), which ensures that the nominal control law $\bar{u}_{k}-K\left(x-\bar{x}_{k}\right)$ is feasible for any $x$ in the scaled and translated terminal set, force us to perform a possibly expensive vertex enumeration of the terminal set $\mathcal{T}$. Let $\mathcal{T}$ have $\nu_{p}$ vertices $v_{j}$ and it follows that $\lambda_{k} \mathcal{T}\left(\bar{x}_{k}\right)$ can be represented as the convex hull $\operatorname{conv}\left(\bar{x}_{k}+\lambda_{k} v_{j}\right)$. By convexity, this polytope is in $\mathcal{X}$ if and only if all vertices are. With $\mathcal{X}=\left\{x \mid F_{\mathcal{X}} x \leq b_{\mathcal{X}}\right\}$ we arrive at $F_{\mathcal{X}}\left(\bar{x}_{k}+\lambda_{k} v_{j}\right) \leq b_{\mathcal{X}}$. Similarly for the control constraints, we have to ensure that $F_{\mathcal{U}}\left(\bar{u}_{k}-K\left(x-\bar{x}_{k}\right)\right) \leq b_{\mathcal{U}}$ for all $x \in \operatorname{conv}\left(\bar{x}_{k}+\lambda_{k} v_{j}\right)$. Checking the vertices leads to $F_{\mathcal{X}}\left(\bar{u}_{k}-\lambda_{k} K v_{j}\right) \leq b_{\mathcal{U}}$. To summarize, all constraints are cast as linear inequalities.

With our reformulations, and the polytopic notation introduced above for $\mathcal{X}, \mathcal{U}$ and $\mathcal{T}$, we arrive at a standard quadratic program.

$$
\begin{aligned}
& \min _{u, \lambda_{k}, \bar{r}_{k}} \mathcal{J}_{k} \\
& \text { s.t. } \\
& x_{k+i+1}=A x_{k+i}+B u_{k+i} \\
& F_{\mathcal{X}} x_{k+i} \leq b_{\mathcal{X}} F_{\mathcal{U}} u_{k+i} \leq b_{\mathcal{U}} \\
& F_{\mathcal{T}}\left(x_{k+N}-\bar{x}_{k}\right) \leq \lambda_{k} b_{\mathcal{T}} \\
& F_{\mathcal{U}}\left(\bar{u}_{k}-K \lambda_{k} v_{j}\right) \leq b_{\mathcal{U}} \forall j=1, \ldots, \nu_{p} \\
& F_{\mathcal{X}}\left(\bar{x}_{k}+\lambda_{k} v_{j}\right) \leq b_{\mathcal{X}} \forall j=1, \ldots, \nu_{p} \\
& F_{\mathcal{X}}\left(\Pi_{x} \bar{r}_{k}\right) \leq(1-\epsilon) b_{\mathcal{X}}, \epsilon>0 \\
& F_{\mathcal{U}}\left(\Pi_{u} \bar{r}_{k}\right) \leq(1-\epsilon) b_{\mathcal{U}}, \quad \epsilon>0
\end{aligned}
$$

The properties of the proposed algorithm can be summarized in the following theorem.

Theorem 1: For any feasible initial state $x_{0}$, the MPC algorithm (10) remains feasible and stabilizes the system (3). Additionally, $x_{k}$ asymptotically converges to a setpoint given by the least squares projection of the reference $r$ onto the ( $\epsilon$-contracted) feasible set.

\section{Proof of Theorem 1}

In this section we prove recursive feasibility, state convergence and properties of the steady state. The proof of recursive feasibility and convergence of the state to a stationary point are straightforward and follow standard proofs found in the literature. In a second step, we show that the setpoint to which the state converges is the setpoint associated with the given reference $r$, if feasible, or the setpoint corresponding to the closest possible reference in a least squares sense.

\section{A. Recursive Feasibility}

Let $\mathcal{X}_{N}$ be the set of $x$ where (10) is feasible. Assume $x_{k} \in \mathcal{X}_{N}$ with an optimal solution given by the sequence $\mathbf{u}^{*}=\left\{u_{k}^{*}, u_{k+1}^{*}, \ldots, u_{k+N-1}^{*}\right\}$ and $\lambda_{k}^{*}$ and $\bar{r}_{k}^{*}$, with predicted state trajectory $\mathbf{x}^{*}=\left\{x_{k+1}^{*}, x_{k+2}^{*}, \ldots, x_{k+N}^{*}\right\}$.

At the next time step $\hat{\mathbf{u}}=\left\{u_{k+1}^{*}, u_{k+2}^{*}, \ldots, u_{k+N-1}^{*}, \bar{u}_{k}^{*}-\right.$ $\left.K\left(x_{k+N}^{*}-\bar{x}_{k}^{*}\right)\right\}$ is a feasible control sequence, since $\bar{u}_{k}^{*}-$ $K\left(x_{k+N}^{*}-\bar{x}_{k}^{*}\right)$ is feasible according to (10f). Furthermore, we use $\lambda_{k+1}=\lambda_{k}^{*}$ and $\bar{r}_{k+1}=\bar{r}_{k}^{*}$. Keeping $\lambda_{k+1}$ and $\bar{r}_{k+1}$ unchanged means that we keep the scaled and translated terminal set unchanged. The new state sequence is $\hat{\mathbf{x}}=$ $\left\{x_{k+2}^{*}, x_{k+3}^{*}, \ldots, x_{k+N}^{*}, x_{k+N+1}\right\}$ where $x_{k+N+1}-\bar{x}_{k}^{*}=$ $(A-B K)\left(x_{k+N}^{*}-\bar{x}_{k}^{*}\right)$. Since $\lambda_{k}^{*} \mathcal{T}$ is positively invariant w.r.t the system $x_{k+1}=(A-B K) x_{k}$ according to Lemma 
1, it follows that $x_{k+N+1}-\bar{x}_{k}^{*}$ stays in $\lambda_{k}^{*} \mathcal{T}$, i.e., the terminal state constraint $x_{k+N+1} \in \lambda_{k}^{*} \mathcal{T}\left(\bar{x}_{k}^{*}\right)$ is satisfied. Since $\lambda_{k}^{*} \mathcal{T}\left(\bar{x}_{k}^{*}\right) \subseteq \mathcal{X}$, state constraints are trivially satisfied.

\section{B. Convergence of state and control}

Now assume that we have an optimal solution at time $k$ and denote the optimal cost $\mathcal{J}_{k}^{*}$. Applying the control sequence $\hat{\mathbf{u}}$ defined in the previous section gives the suboptimal cost as derived below in (11)

It thus follows that the suboptimal cost is

$$
\mathcal{J}_{k+1}=\mathcal{J}_{k}^{*}-\left\|x_{k}^{*}-\bar{x}_{k}^{*}\right\|_{Q}^{2}-\left\|u_{k}^{*}-\bar{u}_{k}^{*}\right\|_{R}^{2}
$$

which implies

$$
\mathcal{J}_{k+1}^{*} \leq \mathcal{J}_{k+1} \leq \mathcal{J}_{k}^{*}-\left\|x_{k}^{*}-\bar{x}_{k}^{*}\right\|_{Q}^{2}-\left\|u_{k}^{*}-\bar{u}_{k}^{*}\right\|_{R}^{2}
$$

In other words, $\mathcal{J}_{k}^{*}$ is strictly decreasing as long as $x_{k}^{*} \neq \bar{x}_{k}^{*}$ and $u_{k}^{*} \neq \bar{u}_{k}^{*}$. Hence $x_{k}^{*} \rightarrow \bar{x}_{k}^{*}$ and $u_{k}^{*} \rightarrow \bar{u}_{k}^{*}$. Note that in the limit we have, since $\bar{x}_{k}^{*}$ and $\bar{u}_{k}^{*}$ represent a stationary pair, that $\bar{x}_{k}^{*}=x_{k}^{*}=x_{k+1}^{*}=\bar{x}_{k+1}^{*}$, i.e., the pseudo setpoint converges too. Furthermore the optimal cost $\mathcal{J}_{k}^{*} \rightarrow\left\|\bar{r}_{k}-r\right\|_{W}^{2}$.

\section{Convergence of pseudo reference}

To show convergence of $\bar{r}_{k}^{*} \rightarrow r_{\perp}$, assume that the system has settled at a setpoint given by $\bar{x}_{k}^{*}, \bar{u}_{k}^{*}$, defined by $\bar{r}_{k}^{*}$. The proof will proceed by contradiction, so we assume $\bar{r}_{k}^{*} \neq r_{\perp}$. Consider a perturbation $(0 \leq \gamma<1)$ of the pseudo reference $\bar{r}_{k}^{*}$ towards $r_{\perp}$, given by

$$
\bar{r}_{\gamma}=\gamma \bar{r}_{k}^{*}+(1-\gamma) r_{\perp}
$$

Our first step is to show that this choice is feasible for $\gamma$ sufficiently close to 1 . By convexity, $\bar{r}_{\gamma}$ is feasible with respect to (10h) and (10i). We use the control sequence
$u_{k+i}=\bar{u}_{\gamma}=\Pi_{u} \bar{r}_{\gamma}$ (which also is feasible by convexity) and the predicted states evolve according to

$$
\begin{aligned}
x_{k+1}= & A \bar{x}_{k}^{*}+B \bar{u}_{\gamma} \\
= & A \bar{x}_{k}^{*}+\gamma B \bar{u}_{k}^{*}+(1-\gamma) B u_{\perp} \\
= & A\left(\gamma \bar{x}_{k}^{*}+(1-\gamma) x_{\perp}+\bar{x}_{k}^{*}-\gamma \bar{x}_{k}^{*}-(1-\gamma) x_{\perp}\right) \\
& +\gamma B \bar{u}_{k}^{*}+(1-\gamma) B u_{\perp} \\
= & \gamma\left(A \bar{x}_{k}^{*}+B \bar{u}_{k}^{*}\right)+(1-\gamma)\left(A x_{\perp}+B u_{\perp}\right) \\
& +(1-\gamma) A\left(\bar{x}_{k}^{*}-x_{\perp}\right) \\
= & \gamma \bar{x}_{k}^{*}+(1-\gamma) x_{\perp}+(1-\gamma) A\left(\bar{x}_{k}^{*}-x_{\perp}\right) \\
= & \bar{x}_{\gamma}+(1-\gamma) A\left(\bar{x}_{k}^{*}-x_{\perp}\right)
\end{aligned}
$$

Applying the same manipulations recursively leads to

$$
x_{k+i}=\bar{x}_{\gamma}+(1-\gamma) A^{i}\left(\bar{x}_{k}^{*}-x_{\perp}\right)
$$

For $\gamma$ sufficiently close to 1 all predicted states are feasible w.r.t $\mathcal{X}$, since $\bar{x}_{k}^{*}$ and $x_{\perp}$ (and thus $\bar{x}_{\gamma}$ ) are strictly inside $\mathcal{X}$ and $(1-\gamma) A^{i}\left(\bar{x}_{k}^{*}-x_{\perp}\right)$ approaches 0 as $\gamma$ goes to 1 . What remains to show is that we can select $\lambda_{k}$ such that $x_{k+N+1} \in \lambda_{k} \mathcal{T}\left(\bar{r}_{\gamma}\right)$ and $\lambda_{k} \mathcal{T}\left(\bar{r}_{\gamma}\right) \subseteq \mathcal{X}$.

Since the pseudo reference is in the strict interior (defined by $\epsilon$ ), it immediately follows that there exist a constant $\epsilon_{\lambda}>0$, determined by the geometry of $\mathcal{T}, \mathcal{X}, \mathcal{U}$ and $\epsilon$, such that $\epsilon_{\lambda} \mathcal{T}(\bar{x}) \subseteq \mathcal{X}$ for any strictly feasible $\bar{x}$. Let $d$ denote the radius of the largest possible Euclidean ball centered at the origin which can be inscribed in $\mathcal{T}$ (i.e., $\operatorname{dist}(0, \partial \mathcal{T})$ ). Since $\mathcal{T}$ contains 0 in its interior by assumption, $d>$ 0 . The distance from the terminal state $x_{k+N+1}$ to the new pseudo setpoint is given by $\left\|x_{k+N+1}-\bar{x}_{\gamma}\right\|=(1-$ $\gamma)\left\|A^{N+1}\left(\bar{x}_{k}^{*}-x_{\perp}\right)\right\|$. If this distance is smaller than $\lambda_{k} d$, the terminal state is inside the scaled and translated terminal set. Hence, if $\gamma \geq 1-\frac{\epsilon_{\lambda} d}{\left\|A^{N+1}\left(\bar{x}_{k}^{*}-x_{\perp}\right)\right\|}$ the terminal state constraint is fulfilled. Since $\mathcal{X}$ is polytopic, the denominator in the expression has an upper bound.

$$
\begin{aligned}
\mathcal{J}_{k+1}= & \sum_{i=0}^{N-1}\left(\left\|x_{k+1+i}-\bar{x}_{k}^{*}\right\|_{Q}^{2}+\left\|u_{k+1+i}-\bar{u}_{k}^{*}\right\|_{R}^{2}\right)+\left\|x_{k+1+N}-\bar{x}_{k}^{*}\right\|_{P}^{2}+\left\|\bar{r}_{k}^{*}-r\right\|_{W}^{2} \\
= & \left\|x_{k}^{*}-\bar{x}_{k}^{*}\right\|_{Q}^{2}+\left\|u_{k}^{*}-\bar{u}_{k}^{*}\right\|_{R}^{2}+\left\|x_{k+N}^{*}-\bar{x}_{k}^{*}\right\|_{P}^{2}+\sum_{i=0}^{N-2}\left(\left\|x_{k+1+i}^{*}-\bar{x}_{k}^{*}\right\|_{Q}^{2}+\left\|u_{k+1+i}^{*}-\bar{u}_{k}^{*}\right\|_{R}^{2}\right) \\
& +\left\|x_{k+N}^{*}-\bar{x}_{k}^{*}\right\|_{Q}^{2}+\left\|\bar{u}_{k}^{*}-K\left(x_{k+N}-\bar{x}_{k}^{*}\right)-\bar{u}_{k}^{*}\right\|_{R}^{2}+\left\|x_{k+1+N}-\bar{x}_{k}^{*}\right\|_{P}^{2}+\left\|\bar{r}_{k}^{*}-r\right\|_{W}^{2} \\
& -\left\|x_{k}^{*}-\bar{x}_{k}^{*}\right\|_{Q}^{2}-\left\|u_{k}^{*}-\bar{u}_{k}^{*}\right\|_{R}^{2}-\left\|x_{k+N}^{*}-\bar{x}_{k}^{*}\right\|_{P}^{2} \\
= & \underbrace{\sum_{i=0}^{N-1}\left(\left.\left\|x_{k+i}^{*}-\bar{x}_{k}^{*}\right\|\right|_{Q} ^{2}+\left\|u_{k+i}^{*}-\bar{u}_{k}^{*}\right\|_{R}^{2}\right)+\left\|x_{k+N}^{*}-\bar{x}_{k}^{*}\right\|_{P}^{2}+\left\|\bar{r}_{k}^{*}-r\right\|_{W}^{2}}_{\mathcal{J}_{k}^{*}} \\
& \underbrace{}_{=\left\|x_{k+N}^{*}-\bar{x}_{k}^{*}\right\|_{Q}^{2}+\left\|K\left(x_{k+N}^{*}-\bar{x}_{k}^{*}\right)\right\|_{R}^{2}+\left\|x_{k+1+N}-\bar{x}_{k}^{*}\right\|_{P}^{2}-\left\|x_{k+N}^{*}-\bar{x}_{k}^{*}\right\|_{P}^{2}} \\
& -\left\|x_{k}^{*}-\bar{x}_{k}^{*}\right\|_{Q}^{2}-\left\|u_{k}^{*}-\bar{u}_{k}^{*}\right\|_{R}^{2} \quad
\end{aligned}
$$




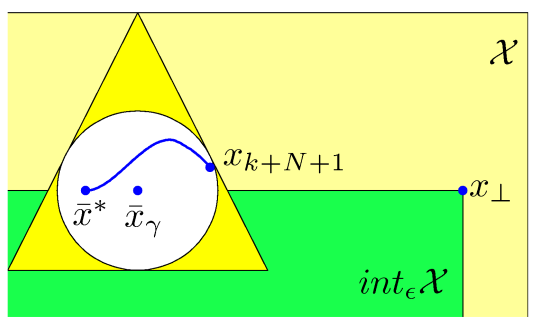

Fig. 1. Illustration of the components in the proof of convergence of the pseudo reference. The figure shows portions of the sets $\mathcal{X}$ and $\operatorname{int}_{\epsilon}(\mathcal{X})$, and the triangular set $\lambda_{k} \mathcal{T}\left(\bar{x}_{\gamma}\right)$ with its inscribed Euclidean ball.

Returning back to the objective function for our proposed feasible solution, and using the notation $\Psi_{i}=A^{i}\left(\bar{x}_{k}^{*}-x_{\perp}\right)$, we arrive at

$$
\begin{aligned}
\mathcal{J}_{k}= & \left\|(1-\gamma) \Psi_{N}\right\|_{P}^{2} \\
& +\sum_{i=0}^{N-1}\left\|(1-\gamma) \Psi_{i}\right\|_{Q}^{2}+\left\|\gamma \bar{r}_{k}^{*}+(1-\gamma) r_{\perp}-r\right\|_{W}^{2} \\
= & (1-\gamma)^{2}\left\|\Psi_{N}\right\|_{P}^{2} \\
& +(1-\gamma)^{2} \sum_{i=0}^{N-1}\left\|\Psi_{i}\right\|_{Q}^{2}+\left\|\gamma\left(\bar{r}_{k}^{*}-r_{\perp}\right)+\left(r_{\perp}-r\right)\right\|_{W}^{2}
\end{aligned}
$$

Differentiate $\mathcal{J}_{k}$ with respect to the step size $\gamma$

$$
\begin{aligned}
\frac{\partial \mathcal{J}_{k}}{\partial \gamma}= & -2(1-\gamma)\left(\left\|\Psi_{N}\right\|_{P}^{2}+\sum_{i=0}^{N-1}\left\|\Psi_{i}\right\|_{Q}^{2}\right) \\
& +2\left(\bar{r}_{k}^{*}-r_{\perp}\right)^{T} W\left(\gamma\left(\bar{r}_{k}^{*}-r_{\perp}\right)+\left(r_{\perp}-r\right)\right)
\end{aligned}
$$

Evaluating this at $\gamma=1$ gives

$$
\left.\frac{\partial \mathcal{J}_{k}}{\partial \gamma}\right|_{\gamma=1}=\left(\bar{r}_{k}^{*}-r_{\perp}\right)^{T} 2 W\left(\bar{r}_{k}^{*}-r\right)
$$

If this inner product is positive it means that the cost function decrease as $\gamma$ decreases which in turn implies that the cost can be reduced by moving $\bar{r}_{k}^{*}$ closer to $r_{\perp}$. Let $W=S^{T} S$ and rewrite the inner product as

$\left(S\left(\bar{r}_{k}^{*}-r_{\perp}\right)\right)^{T}\left(S\left(\bar{r}_{k}^{*}-r\right)\right)=\left\|S\left(\bar{r}_{k}^{*}-r_{\perp}\right)\right\|\left\|S\left(\bar{r}_{k}^{*}-r\right)\right\| \cos \phi$

where $\phi$ is the angle between the vectors. Using the law of cosine and some geometry we can rewrite this into

$$
\begin{aligned}
& \left\|S\left(\bar{r}_{k}^{*}-r_{\perp}\right)\right\|\left\|S\left(\bar{r}_{k}^{*}-r\right)\right\| \cos \phi \\
& =\frac{1}{2}\left\|S\left(\bar{r}_{k}^{*}-r_{\perp}\right)\right\|^{2}+\left\|S\left(\bar{r}_{k}^{*}-r\right)\right\|^{2}-\left\|S\left(r_{\perp}-r\right)\right\|^{2} \\
& =\underbrace{\frac{1}{2}\left\|\bar{r}_{k}^{*}-r_{\perp}\right\|_{W}^{2}+\underbrace{\left\|\bar{r}_{k}^{*}-r\right\|_{W}^{2}-\left\|r_{\perp}-r\right\|_{W}^{2}}_{>0}}_{>0}
\end{aligned}
$$

Since $r_{\perp}$ by definition is the closest feasible point to $r$ in the chosen norm, the right hand side is strictly greater than zero unless $\bar{r}_{k}^{*}=r_{\perp}$. This means that $\mathcal{J}_{k}$ can be improved by making an arbitrarily small move towards $r_{\perp}$ and hence, the solution cannot converge to $\bar{r}_{k}^{*}$ unless $\bar{r}_{k}^{*}=r_{\perp}$. Since we know that the solution converges, it follows that it converges to $\bar{r}_{k}^{*}=r_{\perp}$. To conclude $x_{k} \rightarrow \bar{x}_{k}^{*}=\Pi_{x} \bar{r}_{k}^{*}, u_{k} \rightarrow \bar{u}_{k}^{*}=$ $\Pi_{u} \bar{r}_{k}^{*}$ and $\bar{r}_{k}^{*} \rightarrow r_{\perp}$, and the optimal cost converges to $\mathcal{J}^{*}=$ $\left\|r_{\perp}-r\right\|_{W}^{2}$

\section{EXAMPLE}

A motivating example is taken from the aircraft industry. When maneuvering an aircraft the pilot commands a change in the so called angle of attack, $\alpha$, i.e., the angle between the speed vector and the aircraft $\mathrm{x}$-axis. A large angle of attack means a fast turn rate but if the angle becomes too large the airflow around the wing loses its lifting force and the aircraft stalls. For an agile fighter aircraft superior manoeuvrability is vital for its success in any mission, e.g., avoiding enemy air defence missiles or outmaneuver hostile aircrafts. Therefore one wants to be able to control the angle of attack to the limit of what is possible, but not to a value so big that the aircraft loses lift force and stalls.

The modern concept of carefree maneuvering means that the pilot shall be able to entirely focus on the mission tasks while the flight control system automatically limits the attainable angles and velocities so that the aircraft remains controllable no matter the pilot's inputs (so called maneuver load limits).

The equations of motion describing this can be formed as a two state dynamical system with the angle of attack, $\alpha$, as the fist state and the pitch rate, $q$, as the second, the so called short period dynamics, see [13].

In this example we consider a linearized form of the unstable short period dynamics for a fighter aircraft, discretized using a sample-time of $60 \mathrm{~ms}$.

$$
\begin{aligned}
{\left[\begin{array}{c}
\alpha_{k+1} \\
q_{k+1}
\end{array}\right] } & =\underbrace{\left[\begin{array}{ll}
0.9719 & 0.0155 \\
0.2097 & 0.9705
\end{array}\right]}_{A}\left[\begin{array}{c}
\alpha_{k} \\
q_{k}
\end{array}\right]+\underbrace{\left[\begin{array}{l}
0.0071 \\
0.3263
\end{array}\right]}_{B} \delta_{k} \\
y & =\underbrace{\left[\begin{array}{ll}
1 & 0
\end{array}\right]}_{C}\left[\begin{array}{c}
\alpha_{k} \\
q_{k}
\end{array}\right]
\end{aligned}
$$

The maneuver load limits for angle of attack and pitch rate has been set to

$$
x_{k} \in \mathcal{X}=\left\{(\alpha, q)^{T} \mid-15 \leq \alpha \leq 30,-100 \leq q \leq 100\right\}
$$

The elevator angle deflection has been limited to $25^{\circ}$

$$
\delta_{k} \in \mathcal{U}=\{\delta \mid-25 \leq \delta \leq 25\}
$$

The objective is to have $\alpha$ track a reference $r$, possibly out to the boundary of the feasible set $\mathcal{X}$.

The weighting matrices in (8) have been chosen as $Q=$ $\left[\begin{array}{cc}10 & 0 \\ 0 & 1\end{array}\right], R=1, W=10^{4}$ and $P$ is the Lyapunov cost of the corresponding LQ controller. The prediction horizon is chosen to $N=20$ and $\epsilon$ is chosen small enough to not have any noticable effect, $\epsilon=10^{-5}$.

The sets $\mathcal{X}$ and $\mathcal{T}$ are shown in Figure (2). In this example, we have a scenario where the invariant set extends over the whole feasible region in the $\alpha$-direction. Hence, the invariant set cannot be translated at all along the $\alpha$-direction. Since $\alpha$ is the variable which should track a reference, thus forcing 
us to place the invariant set at a coordinate satisfying $\alpha=r$, scaling is absolutely necessary.

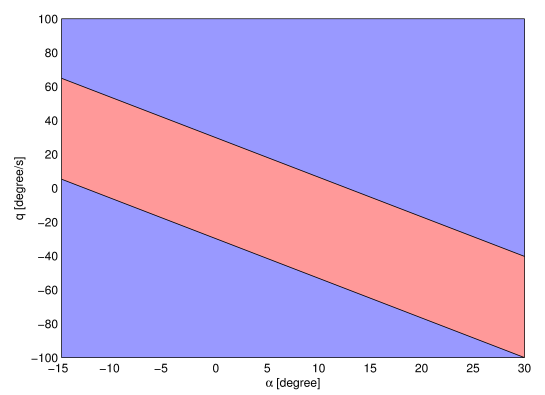

Fig. 2. The feasible set $\mathcal{X}$ (blue) and the positively invariant terminal set $\mathcal{T}$ (red). The invariant set extends over the whole feasible region in the $\alpha$-direction. Hence, the invariant set cannot be translated at all in this direction.

The implementation and simulation has been performed in MATLAB with YALMIP [14] and MPT [15] toolboxes.
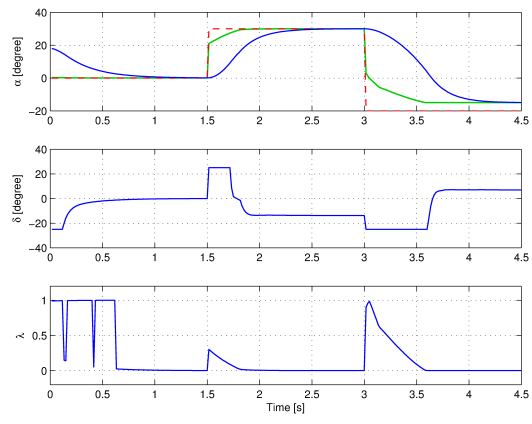

Fig. 3. The upper figure shows the pilot input reference, $r$ (red dashed), the pseudo reference $\bar{r}$ (green) and the output $\alpha$ (blue). The middle figure shows the control signal and the bottom figure shows the change of the scaling variable $\lambda$ over time.

A time plot of the simulation is shown in figure 3. It can be seen that the admissible reference $\bar{r}_{k}$ is a prefiltered version of the pilots reference input $r$, and that both $\bar{r}_{k}$ and $\alpha$ converges to the desired reference if feasible. When setting the reference to $\alpha=30^{\circ}$, i.e., when it is located on the border of $\mathcal{X}$ the output will track the reference, but when the reference is set to $\alpha=-20^{\circ}$, i.e., outside the feasible set, the output will track the pseudo reference that converge to the closest feasible point.

The variations in $\lambda_{k}$ when $\bar{r}_{k}=0$ during the first 1.5 seconds simply illustrate the fact that $\lambda_{k}$ can be non-unique. After half a second or so, the predictions reach the origin without constraining them to do so. Hence, any value of $\lambda_{k}$ between zero and one will be feasible, and the value returned depends on the algorithm used to solve the quadratic program. Note that $\lambda_{k}$ in no sense is used in the actual control input, hence the chattering in $\lambda_{k}$ does not transfer to $u_{k}$. At $t=1.5$ seconds a change in the reference is made, and the algorithm increases $\lambda_{k}$ slightly to retain feasibility to a new pseudo setpoint. After a while the scaling approaches zero, this time not an arbitrary choice, but a necessity since the pseudo setpoint approaches the border of $\mathcal{X}$. Finally, a large change in the reference is made after 3 seconds. The algorithm counter-acts with a large pseudo reference change to roughly the origin, while inflating the terminal set maximally. Following this large change, the pseudo reference slowly moves out to the border $-15^{\circ}$ while shrinking the terminal set.

\section{CONCLUSIONS}

An extension to the standard MPC methods to allow for tracking setpoints arbitrary close to the boundary of the admissible state space has been presented.

It has been proven that by scaling the terminal state constraint set with a positive scalar $\lambda$ and requiring that the scaled and translated terminal set is a subset of the feasible set, both stability and recursive feasibility can be guaranteed for setpoints that approach the boundary of the feasible set.

The theory and its performance has been illustrated with an example from the aeronautical industry. The examples shows that when the reference is set to the limit of the states the invariant set is scaled accordingly and the system converge to the closest feasible point to the reference.

Acknowledgement: This work has been done as a cooperation between Linköping University and Saab Aeronautics and is funded by VINNOVA and CENIIT

\section{REFERENCES}

[1] D. Mayne, J. Rawlings, C. Rao, and P. Scokaert, "Constrained model predictive control: Stability and optimality," Automatica, vol. 36, no. 6, pp. 789-814, Jun. 2000.

[2] K. R. Muske and J. B. Rawlings, "Model predictive control with linear models," AIChE Journal, vol. 39, no. 2, pp. 262-287, Feb. 1993.

[3] K. R. Muske and T. Badgwell, "Disturbance modeling for offset-free linear model predictive control," Journal of Process Control, vol. 12, no. 5, pp. 617-632, Aug. 2002.

[4] G. Pannocchia and E. C. Kerrigan, "Offset-free Receding Horizon Control of Constrained Linear Systems subject to Time-varying Setpoints and Persistent Unmeasured Disturbances," University of Pisa, Tech. Rep., 2003.

[5] G. Pannocchia and J. B. Rawlings, "Disturbance models for offset-free model-predictive control," AIChE Journal, vol. 49, no. 2, pp. 426-437, Feb. 2003.

[6] D. Limon, I. Alvarado, T. Alamo, and E. Camacho, "MPC for tracking piecewise constant references for constrained linear systems," Automatica, vol. 44, no. 9, pp. 2382-2387, Sep. 2008.

[7] A. Ferramosca, D. Limon, I. Alvarado, T. Alamo, and E. Camacho, "MPC for tracking with optimal closed-loop performance," Automatica, vol. 45, no. 8, pp. 1975-1978, Aug. 2009.

[8] U. Maeder and M. Morari, "Offset-free reference tracking for predictive controllers," 2007 46th IEEE Conference on Decision and Control, pp. 5252-5257, 2007.

[9] 1 , "Offset-free reference tracking with model predictive control," Automatica, vol. 46, no. 9, pp. 1469-1476, Sep. 2010.

[10] A. Rossiter, "A GLOBAL APPROACH TO FEASIBILITY IN LINEAR MPC," in UKACC ICC, 2005.

[11] C. V. Rao and J. B. Rawlings, "Steady states and constraints in model predictive control," AIChE Journal, vol. 45, no. 6, pp. 1266-1278, Jun. 1999.

[12] F. Blanchini, "Set invariance in control," Automatica, vol. 35, pp. 1747 - 1767, 1999.

[13] B. L. Stevens and F. L. Lewis, Aircraft Control and Simulation, 2nd ed. John Wiley \& Sons, 2003.

[14] J. Löfberg, "YALMIP: A Toolbox for Modeling and Optimization in MATLAB," in Proceedings of the CACSD Conference, Taipei, Taiwan, 2004. [Online]. Available: http://users.isy.liu.se/johanl/yalmip

[15] M. Kvasnica, P. Grieder, and M. Baotic, "Multi-Parametric Toolbox (MPT)," Hybrid Systems: Computation and Control, pp. 121 - 124, 2004. 